MLS PSYCHOLOGY RESEARCH

https://www.mlsjournals.com/Psychology-ResearchJournal

\title{
LA OTREDAD, RESOLUCIÓN DE CONFLICTOS Y MEDIACIÓN SOCIAL: HERRAMIENTAS REFLEXIVAS ANTE LA DIVERSIDAD DE PENSAMIENTO POLÍTICO EN LÍDERES ESTUDIANTILES UNIVERSITARIOS
}

\author{
Jinette Gabriela Labrador Fernández \\ Universidad del Zulia (Venezuela) \\ jinettelabrador@gmail.com·http://orcid.org/0000-0002-9475-6524 \\ Juana Cecilia Ojeda de López \\ Universidad del Zulia (Venezuela) \\ juanacecilia@hotmail.com \\ Gabriel Argota \\ Universidad del Zulia (Venezuela) \\ gabrielargota@gmail.com
}

\begin{abstract}
Resumen. La investigación sobre otredad, resolución de conflictos y mediación social, surgió ante el agravante de discusiones, que la mayoría de las veces culminaron en violencia física y verbal, entre los estudiantes con vida política activa en las universidades venezolanas. Ésta se desarrolló en el contexto educativo universitario de la Facultad de Arquitectura y Diseño de la Universidad del Zulia, Venezuela; por lo que, el objetivo general consistió en analizar las relaciones interpersonales de los educandos que desarrollan un quehacer político en la FAD-LUZ. El marco metodológico se estructuró con un enfoque cuantitativo, desde una tipología causal, con un diseño no experimental transeccional. La muestra se tomó de la población que integra el Centro de Estudiante de la FAD-LUZ. Los principales hallazgos dieron a conocer que la negación del otro ante la diversidad de pensamiento en la dimensión política genera relaciones de conflictos manifiestos. Se concluye que la resolución de conflicto con base en la otredad genera el respeto mutuo entre líderes políticos universitarios, construyendo redes de conversaciones hacia el otro, la otra como un legítimo en convivencia con diversidad de pensamientos, resolviendo las diferencias desde el mutuo respeto.
\end{abstract}

Palabras clave: Otredad, Resolución de conflictos, Respeto, Política, Líderes universitarios. 


\title{
THE OTHER, RESOLUTION OF CONFLICTS AND SOCIAL MEDIATION: REFLECTIVE TOOLS TO THE DIVERSITY OF POLITICAL THINKING IN UNIVERSITY STUDENT LEADERS
}

\begin{abstract}
Research on otherness, conflict resolution and social mediation arose from the aggravation of discussions, which most often culminated in physical and verbal violence, among students with active political life in Venezuelan universities. This was developed in the university educational context of the Facultad of Arquitectura and Diseño of the Universidad del Zulia, Venezuela; Therefore, the general objective was to analyze the interpersonal relationships of the students who develop a political task in the FAD-LUZ. The methodological framework was structured with a quantitative approach, from a causal typology, with a non-experimental transectional design. The sample was taken from the population that integrates the Student Center of the FAD-LUZ. The main findings revealed that the denial of the other to the diversity of thought in the political dimension generates relationships of manifest conflicts. It is concluded that the resolution of conflict based on otherness generates mutual respect between university political leaders, building networks of conversations towards the other, the other as a legitimate coexistence with diversity of thoughts, resolving differences from mutual respect.
\end{abstract}

Keywords: Other, Conflict Resolution, Respect, Politics, University Leaders.

\section{Introducción}

El Orientador en el contexto educativo universitario busca herramientas indispensables que le permitan intervenir de manera neutral e imparcial el requerimiento solicitado de la población donde labora, con el propósito de crear en los asesorados(as), la reflexión para que éstos exploren, planifiquen y evalúen su hacer como seres biológicos, dinámicos e integrales. Esto, con la intención de que todo aquel bachiller que busque el servicio de orientación ${ }^{1}$, pueda atender desde el sentir de sus emociones situaciones de conflictos desde el mutuo respeto. Ahora, ¿qué papel juega la otredad, la resolución de conflictos y la mediación social de lo aquí señalado? En primer lugar y, a pesar de todos los esfuerzos por mejorar la calidad de la Educación Universitaria, aún, la mayoría de los educandos enfrentan sus diferencias desde una actitud violenta tanto física como verbal, lo que los lleva a una construcción de sí mismo aislados del sentir de lo humano, la despersonalización de los valores humanos, de la ética, la moral y la filosofía de vida que cada uno teje en sus conversaciones desde el convivir con el otro. El papel fundamental que juega la resolución de conflicto se sustenta en cómo resolver los problemas que se presentan en la cotidianeidad, en la cual, el otro/la otra es un legítimo en convivencia y cada uno tiene una historia de vida, porque conflictos van a devenir del día a día, ya que el ser humano como ente dinámico está en constante movimiento en el mundo y entre seres vivos.

Existe preocupación en muchos profesionales del área de la orientación al observar, en los espacios universitarios, como pareciera imponerse el irrespeto desde la dirigencia estudiantil, como único modo de abordar las discrepancias políticas y sociales de la nación, reflejado en una activa negación a la dinámica relacional de sus miembros, que en algunos casos se llega a la violencia física, psicológica y ecológica, lo que podría desencadenar situaciones de rompimiento estructural social. Entre algunos de los ejemplos, se puede mencionar el evidenciado el viernes 30 de octubre de 2015 mientras

\footnotetext{
${ }^{1}$ La Universidad del Zulia, junto con la Universidad de Carabobo egresan profesionales de la mención de Orientación, quienes se encargan de trabajar el proceso biopsicosocial de los estudiantes en el contexto educativo, desde la etapa inicial hasta la universitaria.
} 
se realizaba la jornada electoral estudiantil en la Facultad de Humanidades y Educación de la Universidad del Zulia, donde pereció un estudiante de derecho ( PANORAMA, 2015). Otro hecho se visualizó el 5 de mayo de 2018 donde cinco estudiantes de uno de los grupos políticos de la Facultad de Ingeniería de LUZ se desplazaron hacia las instalaciones de la Facultad de Arquitectura y Diseño (FAD) sólo para golpear despiadadamente a un joven perteneciente al Centro de Estudiante de la FAD-LUZ, dejándolo inconsciente, por presentar posturas adversas a ellos. Actualmente, el nivel de irrespeto se torna cada vez más elevado.

En consecuencia, ¿qué hace que entre compañeros estudiantiles que vivenconviven en el recinto universitario, manejen las diatribas sobre la situación política y social que viven como venezolanos y venezolanas por medio de conductas de violencia verbal y física? Podría considerarse, de las conversaciones sostenidas entre los líderes estudiantiles, que los mismos se mueven en el descontento, debido al mal manejo de los bienes nacionales administrados por el actual gobierno; en tal sentido, surge la necesidad de pensar que la diversidad de sentires íntimos, haceres y emociones danzan en el ámbito sensorial-operacional-relacional de premisas aceptadas a priori de las ideologías políticas de las que decidieron no dudar, como verdad universal y transcendente, generando así la negación del otro, la otra como un legítimo en convivencia.

De lo antes expuesto, es el momento de fijar la atención en tres dimensiones: la primera, mirar al otro como un legítimo en convivencia, la segunda, enfocar a la resolución de conflictos como un evento natural de la vida en comunidad y tercero, la negociación y mediación como estrategias de intervención ante situaciones difíciles en la dinámica relacional interpersonal, intergrupal e internacional. Para ello, el estudio de la otredad se encaminará bajo la perspectiva del Dr. Humberto Maturana, Dávila Ximena (2015), entre otros, todo esto en función del dominio experiencial para considerar la otredad el punto de partida ante la construcción de un mundo impregnado de valores y principios, para que pueda vivirse la aceptación del otro como un legítimo en convivencia. A la resolución de conflictos, con base en el entendimiento de lo que es el conflicto, la negociación y mediación desde los fundamentos epistemológicos de FUNIBER (s/f), el modelo transformador de Bush y Folger (1996) como herramienta de intervención ante un conflicto.

Con base en lo expuesto, en este artículo se analizaran las relaciones interpersonales de los estudiantes que desarrollan una vida política en la FAD-LUZ, Venezuela, mediante el diseño y la implementación de un taller teórico-práctico con el que ofrecerles una formación sobre la resolución de conflictos, a través de las estrategias de negociación, mediación y la aceptación del otro como un legítimo en convivencia, a fin de buscar un matiz más abarcador en un dominio experiencial donde el otro y/o la otra también tenga lugar y en el cual los estudiantes sean co- constructores de un mundo con él.

\section{Método \\ Diseño \\ Está sustentado en una investigación orientada en el paradigma positivista clásico, que según Hernández, Fernández y Baptista (2014), se apoya en el empirismo en términos de lógica intentando buscar una explicación causal y mecanicista de los fenómenos de la realidad. Con un diseño no experimental transeccional, dado que no se manipulan ningunas de las variables; con un enfoque cuantitativo de tipo causal, al querer determinar la relación entre las variables de trabajo empleadas. Además, se aportó una nueva propuesta de aplicación práctica, dado que se trata del diseño de un taller de formación en resolución de conflictos a líderes estudiantiles que hacen vida política en la Facultad}


de Arquitectura y Diseño de LUZ, a fin de poder mejorar su situación, así como el momento actual que está atravesando Venezuela.

Este estudio tuvo la finalidad, en un primer momento, de impartir el conocimiento sobre las teorías de resolución de conflictos con base en las estrategias de negociación y mediación, considerando a la Otredad, desde la perspectiva del Dr. Humberto Maturana, Dávila Ximena (2015) etc., como un componente influyente al momento de resolver las diferencias entre estudiantes que hacen vida política, en relación a la situación sociopolítica de Venezuela y, en un segundo momento, una vez comprendido ese piso epistémico, al instante de tomar la decisión de afrontar los requerimientos exigidos por la Universidad del Zulia, llevar a la praxis las estrategias de resolución de conflicto dadas.

Por lo que, las hipótesis que se plantearon para esta investigación:

- $\quad H_{0}$ : emplear el principio de otredad en la resolución de los conflictos mejora las relaciones intergrupales de los estudiantes que hacen vida política en la FADLUZ.

- $\quad \mathrm{H}_{1}$ : emplear el principio de otredad en la resolución de conflictos no mejora las relaciones intergrupales de los estudiantes que hacen vida política en la FADLUZ.

\section{Variables}

- Independiente: "Resolución de los Conflictos sociales con base en la aceptación del otro".

- Dependiente: "Las relaciones intergrupales de los estudiantes que hacen vida política en la Facultad de Arquitectura y Diseño de LUZ".

\section{Participantes}

Se tomarán en cuenta para el estudio 20 estudiantes que hacen vida política, pertenecientes al Centro de Estudiantes de la Facultad de Arquitectura y Diseño de la Universidad del Zulia, Venezuela.

A causa de los fuertes disturbios sucedidos en los últimos tiempos, muchos de los participantes no pudieron acudir finalmente en la fecha cuando se celebró el taller de formación, por lo que únicamente se pudo trabajar con un total de $65 \%$ de la muestra, en total 13 estudiantes de los 20 iniciales, de los cuales, 5 eran hombre y 8 mujeres, quienes oscilaban entre 18 a 22 años de edad.

\section{Técnicas e Instrumentos}

En el taller sobre resolución de conflicto se utilizó para el trabajo de la autoconciencia: "Percepciones de sí mismo y hacia el otro"; 2 técnicas, la primera, técnica del Espejo, la cual consistió en la mirada de cómo soy, utilizándose el instrumento tipo Cuestionario con pregunta abierta titulado: "Mirando mi ser", la segunda, será la técnica de Observación, donde los líderes estudiantiles estuvieron atentos al nivel de comportamiento o actitud que presentan frente a los compañeros(as) con una ideología política diferente, utilizándose el instrumento de lista de cotejo titulado: "Mi compañero (a) un (a) legitimo (a) conmigo".

Posterior al implemento de las técnicas e instrumentos anteriormente señalados, se trabajaron las redes de conversaciones de la otredad y resolución de conflictos que manejan los estudiantes, con las técnica: "Lluvia de ideas" y el "Diario de emociones", la lluvia de ideas se generó por medio del instrumento de sondeo de distinciones, el cual tiene tres preguntas abierta, recogiéndose la información que los estudiantes manejan por otredad, resolución de conflictos, y cómo resuelven estos al momento de enfrentar los 
problemas sociopolíticos del país; el diario de emociones, contempla qué emociones se repiten día a día, su origen, y las conversaciones que se manejan en el afloramiento de ese emocionar, acompañado del instrumento tipo cuestionario con pregunta abierta .

Finalmente, por medio de la técnica de sesión profunda y el sociodrama, se trabajó la comprensión del conflicto, su proceso, estrategias de resolución de los mismos, utilizándose para ello el instrumento cartografía conceptual, para conocer, analizar y profundizar las distinciones de conflicto, resolución de éstos, negociación, mediación, entre otros y, para llevar a la praxis lo disertado teóricamente, los líderes estudiantiles realizaron, representaciones teatrales sencillas (sociodrama) donde abordaron una situación del contexto, buscaron generar un aprendizaje en torno a la resolución de conflicto con base en el Modelo Transformativo de Bush y Folger (1996) utilizando el instrumento: "Planeación del Sociodrama".

\section{Análisis de datos}

Para la guia de observación, cuestionario abierto y sondeo de conversaciones, se utilizó el proceso de elección de una unidad constante utilizándose dos técnicas: de escrutinio guiado con el material vinculado a una teoría. Y técnica de procesamiento utilizando la metacodificación, donde se examinan las relaciones entre categorías para descubrir otras potencialmente nuevas, generándose los memos analíticos de cada categoría y su relación con los códigos teóricos de referencias para la investigación.

Llevándose a cabo el procedimiento siguiente:

Se seleccionó determinado número de cuestionarios. pregunta.

Se Observó la frecuencia con que aparece cada respuesta a la

Se eligió las respuestas que se presentan con mayor frecuencia (patrones generales de respuesta).

Se clasificaron las respuestas elegidas en temas, aspectos o rubros, de acuerdo con un criterio lógico.

Se les dio un nombre o título a cada tema, aspecto o rubro (patrón general de respuesta).

Asignándoseles un código a cada patrón general de respuesta.

Respecto a la Cartografía Conceptual y el sociodrama, el procesamiento de análisis se hizo a través del mapa de aprendizaje, el cual determina el nivel de desempeño con base en los indicadores, utilizándose para ello los niveles de dominio: Pre-formal, Receptivo, Resolutivo, Autónomo y Estratégico.

Posteriormente, después de vaciar los datos ofrecidos por las respuestas, estos se analizaron aplicando la estadística descriptiva y la inferencial, con base en frecuencias absolutas y relativas; asimismo se aplicaron las medidas de tendencia central con el objeto de alcanzar datos confiables que permitieron generar resultados estadísticos que posteriormente fueron confrontados con las bases teóricas planteadas en este estudio, cosas que derivaron si son o no la solución al problema, permitiendo dar insumos teóricos para la construcción de las estrategias de resolución de conflictos dados por los mismos líderes políticos de la Universidad del Zulia.

\section{Resultados}

La primera variable "Resolución de los Conflictos sociales con base en la aceptación del otro", se basó en indagar la percepción de los líderes estudiantiles de la 
Facultad de Arquitectura y Diseño de LUZ en Venezuela, en torno al papel que ellos tienen ante la situación política del país, el mismo implicó la toma de consciencia de sí mismo, resultando:

Tabla 1

Percepción de sí mismo(a) y hacia el otro(a)

\begin{tabular}{|c|c|c|c|}
\hline Código & $\begin{array}{ll}\text { Categorías } & \text { (patrones } \\
\text { respuestas } & \text { con } \\
\text { frecuencia de mención) } & \\
\end{array}$ & $\begin{array}{r}\text { o } \\
\text { mayor }\end{array}$ & $\begin{array}{l}\quad \mathrm{N}^{\circ} \text { de } \\
\text { frecuencias } \\
\text { de mención }\end{array}$ \\
\hline 1 & Soy una persona que estudia, lucha, con metas & & 13 \\
\hline \multirow[t]{2}{*}{2} & Amable, alegre, buen amigo(a) & & 7 \\
\hline & Impulsivo(a)-desconfiado(a) & & 8 \\
\hline 3 & Ser profesional, exitoso(a), padre y madre de familia, agradar a Dios & & 13 \\
\hline 4 & Disciplina, dedicación, estudio, confianza en sí mismo & & 13 \\
\hline
\end{tabular}

Nota: tomada de Hernández, Fernández y Baptista (2014)

Los investigadores, visualizan que el 65\% de los líderes estudiantiles se perciben , ante todo, como personas que tienen la fortaleza de ser combatientes y luchadores en las metas que se trazan para llegar a ser profesionales exitosos, pero que también desean a futuro formar una familia y toman consciencia que para lograrlo tienen que hacerlo por medio de la dedicación, el estudio y el creer que sí pueden alcanzar lo que se proponen, aunado, al grado de pertenencia que reflejaron hacia la FAD-LUZ cuando respondieron al ítem ¿Quién soy? "soy estudiante de arquitectura". Sin embargo, llama la atención que en el Ítem ¿Cómo soy? El $40 \%$ con 8 repeticiones o frecuencias mencionaron que "dependiendo de la situación" son impulsivos-impulsivas y desconfiado-desconfiada hacia el otro-otra. Por lo que, los investigadores deducen que ante una situación de conflicto el $40 \%$ responde con conductas impulsivas, que pueden, dependiendo de la forma del conflicto, en caso de ser manifiesto, ser de forma violenta verbal o física.

Posteriormente, se abrió el espacio de reflexión en relación a quien es el otro-otra para cada uno de ellos-ellas, analizándose el concepto de otredad desde el fundamento epistemológico de Maturana y Dávila (2015) en la cual los bachilleres demostraron:

Siendo en la escala de puntuación tipo Likert el mínimo 8 y el máximo 40, para 13 líderes encuestados bajo la modalidad de Escalamiento tipo Likert que constituye el $65 \%$, se obtiene con:

Tabla 2

Escala de puntuación tipo Likert

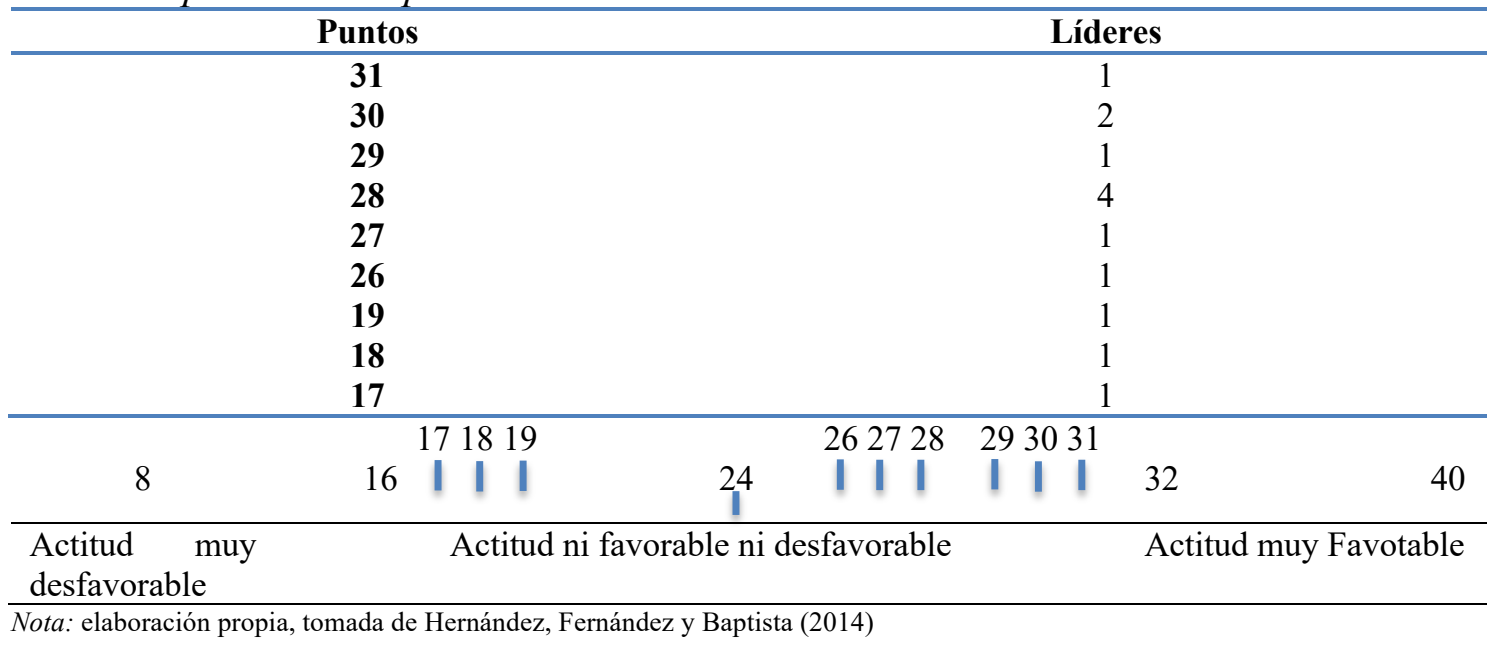


El 65\% de los líderes políticos del Centro de Estudiante de la FAD-LUZ tienen una actitud ni favorable ni desfavorable en relación al otro como un legítimo en convivencia.

Seguidamente, se diagnosticó las redes de conversaciones acerca de la otredad y la resolución de conflictos que manejan los líderes estudiantiles políticos de la facultad de Arquitectura y Diseño de LUZ, donde una vez contractada la distinción de otredad de Maturana y Dávila (2015) con lo que sienten, dicen y hacen los líderes y lideresas con vida política, lograron entender y verbalizar que el otro es un legítimo en convivencia generándose el respeto, donde:

Tabla 3

La otredad y resolución de Conflictos

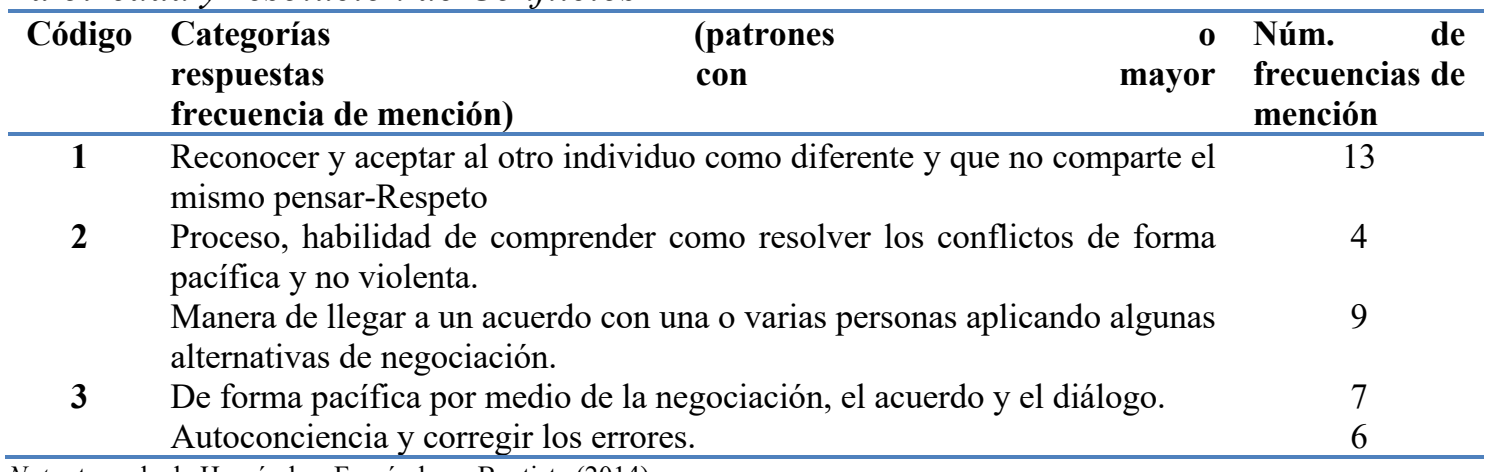

Nota: tomada de Hernández, Fernández y Baptista (2014)

El diagnóstico sobre las verbalizaciones que presentan los jóvenes estudiantes acerca de otredad y resolución de conflictos, una vez contractada con el fundamento epistémico de Maturana, FUNIBER y otros autores, evidencia que el $65 \%$ verbalizó y respondió, que el otro-otra es el reconocer, aceptar y respetar al otro individuo como diferente y que no comparte el mismo pensar.

El 45\% verbalizó y respondió al ítem 2 que la resolución de conflictos es la manera de llegar a un acuerdo con una o varias personas aplicando algunas alternativas de negociación.

Y el 35\% de los líderes estudiantiles respondieron que la forma o el cómo resolverían los conflictos en respuesta al ítem 3, sería de manera pacífica por medio de la negociación, el acuerdo y el diálogo.

Así como también, un aspecto central en tal diagnóstico elevó el nivel del taller en la toma de consciencia de las emociones que están presentes ante las situaciones de conflicto que enfrenta de cara a la divergencia política entre algunos compañeros de clases:

Tabla 4

Emociones presentes en situaciones de conflicto

\begin{tabular}{lllrlr}
\hline Código & $\begin{array}{l}\text { Categorías } \\
\text { respuestas } \\
\text { frecuencia de mención) }\end{array}$ & $\begin{array}{l}\text { (patrones } \\
\text { con }\end{array}$ & $\begin{array}{l}\text { o } \\
\text { mayor }\end{array}$ & $\begin{array}{l}\text { fúm. } \\
\text { frecuencias } \\
\text { mención }\end{array}$ & $\begin{array}{l}\text { de } \\
\text { de }\end{array}$ \\
\hline
\end{tabular}




\begin{tabular}{|c|c|c|}
\hline \multirow[t]{2}{*}{1} & Impotencia, rabia, tristeza. & 12 \\
\hline & $\begin{array}{l}\text { Esperanza, felicidad por el cambio de gobierno en la protesta que no } \\
\text { desmaya y que Dios tiene el control. }\end{array}$ & 1 \\
\hline 2 & $\begin{array}{l}\text { Desánimo, mal humor, discusiones familiares y con amigos. } \\
\text { Aislamiento. }\end{array}$ & 13 \\
\hline \multirow[t]{2}{*}{3} & Rabia, impotencia, ira. & 12 \\
\hline & Esperanza & 1 \\
\hline \multirow[t]{4}{*}{4} & ¿Cuándo será que esto acabará? & 2 \\
\hline & Voluntad de Dios. & 1 \\
\hline & Respeto al otro & 1 \\
\hline & Salida del Presidente-verbalizaciones con palabras obscenas. & 9 \\
\hline
\end{tabular}

Nota: tomada de Hernández, Fernández y Baptista (2014)

Las emociones que subyacen en ellos-ellas ante cómo enfrentan la situación social y política en Venezuela, reflejaron que un $60 \%$ se mueve día a día en la emoción de la impotencia, la rabia y la tristeza. El 65\% tiene acciones o conductas de desánimo para continuar con las actividades académicas, aunado al mal humor, que les lleva a discutir con familiares y amigos, llevándolos al aislamiento que denominaron "encerrase en su cuarto". De modo que, al momento de reaccionar ante situaciones de conflicto inherente a lo político-social el $60 \%$ lo hace desde la rabia, impotencia e ira, manteniendo el $45 \%$ redes de conversaciones obscenas y de rabia pidiendo la salida urgente del Presidente de la República Bolivariana de Venezuela.

En tal sentido, se generó conocer las distinciones de conflictos y clasificar los tipos de conflictos a los que se enfrentan los líderes estudiantiles políticos ante los requerimientos exigidos a la Universidad del Zulia, donde la-el bachiller desarrolló la Cartografía Conceptual arrojando en los niveles del Mapa de Aprendizaje:

Tabla 5

Concepto y Tipos de Conflictos

\begin{tabular}{clcc}
\hline Código & $\begin{array}{l}\text { Categorías (patrones o respuestas con mayor frecuencia de } \\
\text { mención }\end{array}$ & $\begin{array}{l}\text { Número } \\
\text { frecuencias } \\
\text { mención }\end{array}$ & $\begin{array}{l}\text { de } \\
\text { de }\end{array}$ \\
\hline $\mathbf{1}$ & Conflicto, es cuando dos o más personas difieren de sus puntos de & 12 \\
& vista, pero que no es malo cuando se crece emocionalmente. & \\
& La diferencia que se tiene con uno mismo y otras personas. & 1 \\
$\mathbf{2}$ & Intraindividual-interindividual & 2 \\
& Intraindividual & 4 \\
$\mathbf{3}$ & Interindividual & 7 \\
& Latente-manifiesto & 6 \\
$\mathbf{4}$ & Latente & Colectivo universitario & 13 \\
\hline
\end{tabular}

Nota: tomada de Hernández, Fernández y Baptista (2014)

Al contractar el fundamento epistemológico de FUNIBER con el hacer de los estudiantes con vida política en la FAD-LUZ, 60\% de los bachilleres al conocer las distinciones sobre conflicto, lograron conceptualizar el mismo diciendo que ese, es cuando dos o más personas difieren de sus puntos de vista, pero que no es malo cuando se crece emocionalmente. $35 \%$ se identifica dentro de un tipo de conflicto intraindividual, con una forma latente-manifiesta $y$, unánime con $65 \%$ dentro de un ámbito colectivo universitario.

Ya conocido la distinción de conflicto y el tipo de conflicto más vivenciado por ellos-ellas, se especifica el perfil de confrontación ante el conflicto de los líderes estudiantiles que hacen vida política en la Universidad del Zulia. El cual también se 
desarrolló mediante la Cartografía, evidenciándose en los niveles del Mapa de Aprendizaje:

Tabla 6

Perfil de Confrontación ante el Conflicto

\begin{tabular}{clc}
\hline Código & $\begin{array}{l}\text { Categorías (patrones o respuestas con mayor frecuencia } \\
\text { de mención }\end{array}$ & $\begin{array}{l}\text { Número de } \\
\text { frecuencias } \\
\text { de mención }\end{array}$ \\
\hline $\mathbf{1}$ & Evitarlo o Retirarse & 4 \\
$\mathbf{2}$ & Competir, obligar o poder & 3 \\
$\mathbf{3}$ & Adaptación, conciliación o complacer & - \\
$\mathbf{4}$ & Concesión o pacto & 5 \\
$\mathbf{5}$ & Colaboración, cooperación & 1 \\
\hline Nota: tomada de Hernández, Fernández y Baptista (2014)
\end{tabular}

Nota: tomada de Hernández, Fernández y Baptista (2014)

Se evidencia que el $25 \%$ tiene un perfil de confrontación ante el conflicto mediante la concesión y pacto, $20 \%$ prefiere evitar el conflicto y retirarse y $15 \%$ lo resuelve por medio de la competencia y el poder.

Una vez claros para el estudiante lo que es otredad, las verbalizaciones que manejan del otro, el conocer el conflicto como un aspecto natural en el fluir del vivir, los tipo de conflicto más vivenciando por ellos-ellas, el perfil como los confrontan; los bachilleres se plantean cómo resolverlos, de modo que los investigadores propician el espacio psíquico para que conozcan las estrategias de negociación y mediación para la construcción de las relaciones intergrupales con base en la cultura de paz, y con ello, abordar mejorar la relación intergrupal de los estudiantes que hacen vida política en la Facultad de Arquitectura y Diseño de LUZ.

Tales constructos se discutieron mediante la Cartografía Conceptual, arrojando en los niveles del Mapa de Aprendizaje:

Tabla 7

Estrategias de negociación y mediación

\begin{tabular}{clc}
\hline Códigos & \multicolumn{1}{c}{$\begin{array}{c}\text { Categorías (patrones o respuestas con mayor } \\
\text { frecuencia de mención }\end{array}$} & $\begin{array}{c}\text { Número de } \\
\text { frecuencias de mención }\end{array}$ \\
\hline 1 & Charla privada de la estrategia de Negociación & 12 \\
2 & Mediación & 1 \\
\hline Nota: tomada de Hernández, Fernández y Baptista (2014)
\end{tabular}

El $60 \%$ se inclina a resolver los conflictos por medio de la técnica de negociación utilizando la estrategia de sesión privada.

Por último, los bachilleres realizaron un sociodrama donde hicieron una pequeña obra teatral presentando un problema del contexto a resolver, el cual sería desde el Modelo Transformativo de Bush y Folger, evidenciándose en el Mapa de Aprendizaje:

Tabla 8

Transformación en la convivencia. Modelo Transformativo

\begin{tabular}{clc}
\hline Códigos & $\begin{array}{l}\text { Categorías (patrones o respuestas con mayor frecuencia } \\
\text { de mención }\end{array}$ & $\begin{array}{l}\text { Número de frecuencias de } \\
\text { mención }\end{array}$ \\
\hline 1 & Atención a la amistad, a las relaciones de compañerismo & 13 \\
\hline
\end{tabular}




\begin{tabular}{|c|c|c|}
\hline 2 & $\begin{array}{l}\text { Revalorización de cada una de las parte, toma de conciencia } \\
\text { de los involucrados en el protagonismo que tienen y las } \\
\text { emociones que la dirigen. }\end{array}$ & 13 \\
\hline 3 & $\begin{array}{l}\text { Aceptación de reconocer al otro u otra como un legítimo en } \\
\text { convivencia: respeto mutuo y la responsabilidad compartida } \\
\text { en el proceso de resolución de conflictos. }\end{array}$ & 13 \\
\hline 4 & $\begin{array}{l}\text { Redes de conversaciones hacia la colaboración respeto, } \\
\text { dialogo y acuerdos. }\end{array}$ & 13 \\
\hline
\end{tabular}

El 65\%, alcanzó una nueva mirada del otro (“outside”) y de sí mismos (“inside”). Así como también, se trabajaron y lograron los objetivos planteados para este estudio.

\section{Discusión y conclusiones}

La dinámica relacional de violencia física y verbal de cara a la diversidad de la ideología política entre estudiantes, sustentó, en los investigadores, la razón de trabajar con líderes políticos del Centro de Estudiante de la FAD-LUZ, la formación sobre la resolución de conflicto, pero con base en la otredad para que se pueda hablar y hacer relaciones desde el mutuo respeto, debido a que como plantea Maturana y Dávila (2015) el amor es la aceptación del otro como un legítimo en convivencia y que ésta es la emoción que funda lo humano y en la cual la misma se teje y conserva en redes de conversaciones.

En tal sentido, si el estudiante cambia las redes de conversaciones de aspectos negativos del otro por diferir en lo político, cambian las acciones hacia conductas colaborativas desde el mutuo respeto, ya que, como afirma Maturana (2002, p.42):

El "lenguajear" de hecho ocurre en la vida cotidiana entrelazado con el emocionar, y a lo que pasa en este entrelazamiento llamo conversar. Los seres humanos siempre estamos en la conversación, pero el lenguaje, como fenómeno, se da en el operar en coordinaciones de coordinaciones conductuales consensuales recurrentes.

Por lo que, el taller formativo para estos estudiantes sobre la resolución de conflicto les permitió no solo conocer las teorías de otredad y cómo resolver las diversidades entre compañeros mediante estrategias de negociación, mediación y aceptación del otro en su ser legítimo, sino transformar las acciones que le llevan a resolver las diferencias de una forma competitiva de daño moral, psicológico y físico.

Ahora bien, para alcanzar los objetivos propuestos, el estudiante abrió un espacio de reflexión de cómo es que hace lo que hace mediante la autoconsciencia de quién y cómo es, así como también preguntándose quién es el otro o la otra para sí mismo(a), auto-describiendo su sentires íntimos, emociones y formas de hacer, pero también, reconociendo las redes de conversaciones que la sustentan, de este modo se inició un trabajo donde la experiencia de los bachilleres con vida política en la FAD, contractada con la teoría sobre otredad y resolución de conflictos generó las conclusiones siguientes:

1. El percibirse el estudiante mediante la autodescripción de: sus acciones, las consecuencias de éstas y las emociones que subyacen tales conductas, la/el bachiller reflexiona en cómo es que hace lo que hace, desencadenando cambios en el hacer con base en el respeto mutuo.

La reflexión sobre la percepción de los líderes estudiantiles de la facultad de arquitectura y diseño de la Universidad del Zulia en Venezuela, en torno al papel que ellos tienen ante la situación política del país, logró en el estudiante cambio de su sentir íntimo hacia quien difiere de su ideología política, moviéndose en la emoción del respeto 
y proyectando una actitud más interesada a la legitimidad que cada persona forma en la trayectoria histórica del vivir-convivir; esto reflejado en lo que argumentaba Maturana y Varela (2004) "la autoconciencia siempre lleva a un cambio en el curso de las interacciones de los participantes de un proceso de lenguajear en el dominio de autoconciencia..., la conciencia de sí mismo..., es un instrumento para elicitar cambio estructural" (p. 183).

2. Los seres humanos viven en el conversar y, dependiendo de lo que conversan será su proceder y viceversa.

Bajo esa premisa maturanística, los bachilleres iniciaron la formación de las bases epistemológicas sobre otredad y resolución de conflictos, después de discernir acerca de las conversaciones que tejen de sí mismos, el otro, la otra, lo otro, los líderes estudiantiles distinguen por otredad "Reconocer y aceptar al otro individuo como diferente y que no comparte el mismo pensar-Respeto" ((Líderes estudiantiles del Centro de Estudiante de la FAD-LUZ, 2017, a partir de las bases epistemológicas de Maturana y Dávila, 2015).

Y por resolución de conflicto con base en la otredad "es la manera de llegar a un acuerdo con una o varias personas aplicando algunas alternativas de negociación".

Contractar la teoría con la experiencia genera cambios significativos en las redes de conversaciones, desmontando las verbalizaciones que connotan negación del otro-otra por conversaciones que distinguen el respeto a la diversidad ideológica política, estableciéndose relaciones cooperativas y colaborativas.

3. Al conocer los líderes estudiantiles políticos el conflicto y clasificar los tipos de conflictos a los que se enfrentan, los espacios de reflexión llevan a la deconstrucción de configuraciones negativas sobre conflicto, construyendo así redes de conversaciones positivas donde los cambios estructurales internos orientan a conductas menos violentas y más colaborativas y cooperativas con base en el respeto y la aceptación de la diversidad política del otro, la otra.

Así, los jóvenes lograron construir la distinción de conflicto exponiendo: "es cuando dos o más personas difieren de sus puntos de vista, pero que no es malo cuando se crece emocionalmente-respeto" (Líderes estudiantiles del Centro de Estudiante de la FAD-LUZ, 2017, a partir de los conocimientos de FUNIBER, s/f).

El tipo de conflicto más recurrente en los bachilleres del Centro de Estudiante de la FAD-.LUZ es el intraindividual, debido a que persiguen ideologías y objetivos políticos divergentes entre ellos-ellas y otros Centros de Estudiantes.

La forma en que se da el conflicto en la actualidad para estos bachilleres de la FAD-LUZ es latente-manifiesto por la situación de disturbio permanente que viven.

El ámbito más repetido en los líderes de la FAD donde se generan los conflictos es el colectivo universitario, en vista de que la lucha de calle está liderada por los estudiantes de los centros estudiantiles, planificándose dentro del recinto universitario y manifestada en las adyacencias del mismo. Aunque, se lleva a cabo con grupos de ideologías distintas, unos a favor otros en contra.

De esta manera, el bachiller logró entender que el conflicto y/o los conflictos son circunstancias en las que dos o más personas entran en diferencia de puntos de vistas o diatribas, porque sus posiciones, intereses, necesidades, deseos o valores son distinguidos como incompatibles, guiados por las emociones de molestia y donde las coordinaciones de acciones entre las partes en conflicto, pueden ser fortalecidas o deterioradas según cómo sea el proceso de resolución del conflicto.

4. El perfil de confrontación ante el conflicto representa en las personas, en el caso de este estudio, los líderes estudiantiles de la FAD-LUZ, los cambios ontogénicos y filogénicos que tejen en el lenguaje durante la historia del vivir en la deriva natural, constituido por las configuraciones que se entrelazan en la interacción relacional entre las 
estructura dinámica interna y la estructura del medio, generando las distinciones de sí mismos, el otro, la otra, lo otro.

El perfil o los rasgos de confrontación ante el conflicto más recurrentes en los líderes estudiantiles que hacen vida política en la Universidad del Zulia se refleja en 25\% mediante la concesión y pacto, $20 \%$ evita el conflicto y se retira y $15 \%$ lo resuelve por medio de la competencia y el poder.

El líder político de la FAD-LUZ resuelve los conflictos mediante el consenso, acuerdo y diálogo para salir de la crisis política, económica y social que viven, en un $25 \%$.

Hay circunstancia que disparan, en algunos de estos líderes, conductas de violencia, sin embargo, la naturaleza humana en el $25 \%$ de ellos/ellas se inclina a la no violencia, al diálogo y el consenso. Una vez más, los estudiantes ampliaron su experiencia cognitiva al lograr el cuarto objetivo específico identificando el perfil de confrontación ante el conflicto.

5. Conocer los líderes estudiantiles las estrategias de negociación y mediación para la construcción de relaciones intergrupales con base en la cultura de respeto mutuo, ocasionó:

5.1. Aceptar y reconocer al otro $u$ otra como un legítimo en convivencia implicando: el respeto mutuo y la responsabilidad compartida en el proceso de resolución de conflictos.

5.2. Tejer redes de conversaciones hacia la colaboración, respeto, dialogo y acuerdos, generan conductas sin violencia física y verbal.

5.3. Los líderes estudiantiles prefieren resolver los conflictos sociales mediante la estrategia de negociación por medio de sesiones privadas, entre los involucrados y no terceros.

5.4. El modelo de Bush y Folger (1996) en el contexto universitario es una herramienta para la resolución de conflictos con base en la revalorización de la autoestima de los jóvenes y la responsabilidad compartida de las decisiones tomadas y ejecutadas, poniendo énfasis en las relaciones de amistad y compañerismo.

La negociación es un proceso que involucra directamente a las partes, sin necesidad de un tercero, ni de estar frente a una disputa. El día a día trae consigo un constante negociar que lleva a un ganar-ganar a las partes.

Mientras que la mediación involucra un sistema de negociación donde se requiere de una tercera persona imparcial que pueda acompañar a las partes a resolver de manera armónica las diatribas que exponen.

Saber conocer lo que cada una implica, da un abanico de posibilidades que despierta la creatividad en las personas que acuden a estas estrategias para manejar mejor las relaciones y hasta una mejor toma de decisiones.

Los líderes estudiantiles con vida política del Centro de Estudiante de la FADLUZ que entraron al taller de formación sobre resolución de conflicto, no fueron los mismos que salieron en su sentir íntimo, emociones y haceres, ya que, como indica el Modelo transformativo de Bush y Folger (1996, citado por Hernández, 2014):

...consiste, por tanto, en modificar la relación entre las partes, por lo que no se pone tanto énfasis en obtener un acuerdo, aunque por supuesto este sea el objetivo final a alcanzar, pero su logro se pondera como una suerte de consecuencia de esa nueva situación producida entre las partes, como resultado de la transformación producida en la relación existente entre las personas mediadas. El acuerdo sólo se logrará en la medida que los participantes encuentren una nueva mirada del otro ("outside") y de sí mismos (“inside").. (p.71) 
Lo citado por Hernández (2014), haciendo referencia al Modelo de Bush y Folger (1996), produjo "que cada parte potencie su protagonismo y pueda reconocer su cuota de responsabilidad en el desenvolvimiento de la controversia y la que le corresponde a su oponente" (Rivas, 2015, p. 1); esto se evidenció en el Sociodrama que los jóvenes representaron, logrando éstos el último objetivo específico como lo fue el conocer las estrategias de negociación y mediación para la construcción de relaciones intergrupales con base en la cultura de paz, pero además, aplicadas a su experiencia de vida entre compañeros. Asimismo, se obtuvo el trabajar cada uno de ellos, formándose los líderes estudiantiles en la resolución de conflicto mediante las estrategias de negociación, mediación y la aceptación del otro y la otra y, demostrando el logro de todos los objetivos planteados en esta investigación.

Iniciándose el camino para la continua profundización de la resolución de conflictos, que involucra la conservación de la evaluación y cambios que en el tiempo puedan generarse en las estrategias de negociación y mediación con base en la otredad.

Instaurar una cultura de la otredad requiere una mirada de sí mismo, al otro/otra desde la investigación, reflexión, explicación, argumentación y contextualización en el presente cambiante continuo de la deriva natural de las coherencias sensorialesoperacionales-relacionales del ser vivo humano y el nicho ecológico que hace posible ese vivir-convivir.

Finalmente y después de todo lo tratado, se puede afirmar el cumplimiento y alcance del objetivo general propuesto para este estudio que no es otro que el de analizar las relaciones interpersonales de los estudiantes que desarrollan una vida política en la FAD-LUZ, Venezuela, mediante el diseño y la implementación de un taller teóricopráctico mediante el que ofrecerles una formación sobre la resolución de conflictos, a través de las estrategias de negociación, mediación y la aceptación del otro como un legítimo en convivencia, a fin de buscar un matiz más abarcador en un dominio experiencial donde el otro y/o la otra también tenga lugar y en el cual los estudiantes sean co- constructores de un mundo con él. Por ello, podemos concluir con una afirmación y aceptación de una de las hipótesis planteadas al inicio de este estudio en la que se pronosticaba que emplear el principio de otredad en la resolución de los conflictos mejora las relaciones intergrupales de los estudiantes que hacen vida política en la FAD-LUZ.

\section{Referencias}

Estrada, A. (30 de octubre de 2015). Estudiante muerto en LUZ fue empujado contra cartelera de vidrio. PANORAMA. Retrieved from https://www.panorama.com.ve/sucesos/Estudiante-muerto-en-LUZ-fueempujado-contra-cartelera-de-vidrio-20151030-0053.html

Fundación Universitaria Iberoamericana. (2016). Técnicas y mediación en resolución de conflictos. Retrieved from http://panal.funiber.org/alumno/.

Hernández, R., Fernández, C., y Baptista, P. (2014). Metodología de la investigación (4ª Ed.). México: Mc Graw- Hill.

Hernández Ramos, C. (2014). Modelos Aplicables en Mediación Intercultural Barataria. Revista Castellano-Manchega de Ciencias Sociales, (17), 67-80. Retrieved from http://www.redalyc.org/pdf/3221.

Maturana, H. y Dávila, X. (2015). El árbol del vivir. Santiago de Chile: MVP.

Maturana, H y Varela, F. (2004). El Árbol del Conocimiento: las bases biológicas del entendimiento humano ( $1^{\mathrm{a}} \mathrm{ed}$.). Buenos Aires: LUMEN. 
Maturana, H. (2002). Emociones y lenguaje en educación y política. España: OCEANO. Maturana, H. (2002). El sentido de lo humano. España: OCEANO.

Maturana, H. (2002). La objetividad. Un argumento para obligar. España: OCEANO. Maturana, H. y Nisis, S. (2002). Formación humana y capacitación. España: OCEANO. Maturana, H. y Nisis, S. (2002). Transformación en la convivencia. España: OCEANO. Rivas, I. (2015). El Modelo Transformador de Bush y Folger. Recuperado de http://zonaeducacion.worpress.com/2015/05/20/el-modelo-transformativo.

Fecha de recepción: 29/07/2019

Fecha de revisión: 24/08/2019

Fecha de aceptación: 22/12/2019 